\title{
A novel fluorometric aptasensor based on carbon nanocomposite for sensitive detection of Escherichia coli 0157:H7 in milk
}

\author{
Tao Yang, Xinyan Yang, Xiaojie Guo, Shiqian Fu, Jiapeng Zheng, Sihan Chen, $\odot$ Xue Qin, Zhenghui Wang, \\ Dongyan Zhang, Chaoxin Man, * ${ }^{\circ}$ and Yujun Jiang* (i) \\ Key Lab of Dairy Science, Ministry of Education, College of Food Science, Northeast Agricultural University, Harbin, China, 150030
}

\section{ABSTRACT}

Escherichia coli $\mathrm{O} 157: \mathrm{H} 7$ is an extremely serious foodborne pathogen accounting for a vast number of hospitalizations. In this system, a simple, rapid, and safe compound method was developed based on carbonyl iron powder (CIP) and multiwalled carbon nanotubes (MWCNT). Then, the CIP@MWCNT -based aptasensor was constructed by strong $\pi$-stacking between nanocomposite and aptamer, single-strand DNA, causing fluorescent quenching of the dye-labeled aptamer. The restoration of dye fluorescence could be achieved when aptamer came off the surface of the CIP@MWCNT nanocomposite due to the presence of target bacteria. To the best of our knowledge, this fabrication of magnetic carbon nanotubes without irritating and corrosive reagents is described for the first time. The sensing platform was also an improvement on the conventional formation of the aptasensor between carbon materials and DNA aptamer. The nanocomposite was verified by diverse characterization of zeta potential, Fourier-transform infrared spectroscopy, transmission electron microscopy, and energy dispersive x-ray analysis. The CIP@MWCNT-based aptasensor was an effective nanoplatform for quantitative detection of E. coli $\mathrm{O} 157: \mathrm{H} 7$, and was measured to have high specificity, good reproducibility, and strong stability. The aptasensor's capacity to quantify E. coli O157:H7 was as low as $7.15 \times 10^{3} \mathrm{cfu} / \mathrm{mL}$ in pure culture. The detection limit of E. coli O157:H7 was $3.15 \times 10^{2} \mathrm{cfu} /$ $\mathrm{mL}$ in contaminated milk after $1 \mathrm{~h}$ of pre-incubation. Hence, the developed assay is a new possibility for effective synthesis of nanocomposites and sensitive tests of foodborne pathogens in the dairy industry.

Key words: Escherichia coli O157:H7, nanocomposites, aptasensor, milk

\footnotetext{
Received February 11, 2020.

Accepted April 14, 2020.

*Corresponding authors: mcxwh2006@qq.com and yujun_jiang@ 163.com
}

\section{INTRODUCTION}

Foodborne pathogens are a public issue and account for many hospitalizations and deaths each year (https: //www.cdc.gov/foodborneburden/index.html). Of all the foodborne pathogens, Escherichia coli O157:H7 is regarded as the most concerning. Certain efforts for controlling E. coli O157:H7 in food processes have been made, such as abiding by good manufacturing practices, but the effect of prevention is still not obvious (Wu et al., 2015; Zhang et al., 2019). To monitor food safety, microbial culture and molecular-based measurements are used frequently. However, these methods are time consuming, multistep, and require complicated equipment and specialized personnel (Demirkol and Timur, 2016; Li et al., 2018). Thus, establishing a rapid, sensitive, and reliable detection assay is particularly important for reducing the infection of $E$. coli O157:H7 in the dairy industry.

Nanotechnology-based detecting assays are a rapidly extending field that covers various nanomaterials and biomolecules (e.g., aptamer, a low-cost substitute for antibodies targeting antigens specifically). In recent years, these assays have had explosive growth for food safety applications. They have a great capacity for offering superiority over conventional laboratory methods (Krishna et al., 2018). Due to their large surface area, carbon nanotubes have been employed as a carrier to load plentiful biomolecules (such as aptamers) by noncovalent interaction, achieving ultrasensitive detection of targets (Qiu et al., 2015). A nanoplatform was constructed by multiwalled carbon nanotubes (MWCNT) and DNA aptamer according to the $\pi-\pi$ stacking interaction, which was a safe functional strategy that passed cellular toxicity assays (Barbosa et al., 2019). However, the creation of this nanoplatform required several centrifugations to remove excess aptamers, which were cumbersome and time consuming. The operation can be simplified when magnetizing MWCNT is washed in aqueous solution using only a magnetic separator and this sort of treatment has been described several times. The MWCNT nanocomposites with magnetism 
were generally obtained by a solvothermal or coprecipitation method (Deng et al., 2012; Gao et al., 2013; Konni et al., 2017; Wang et al., 2019). Nevertheless, these procedures can be laborious, time consuming, and hazardous due to being multistep (Pourfadakari et al., 2016), using an irritating solution (e.g., ammonium hydroxide; Jamrozik et al., 2014), and requiring a high temperature (Sun et al., 2013). The unique adsorption property of magnetic carbon nanomaterials was widely applied to multiple fields of prevention and control, such as organic acids (Liu et al., 2018), heavy metal ions (Liu et al., 2019), toxins (Wang et al., 2015), and pathogenic bacteria (Khang et al., 2016). An aptasensor was constructed by graphene oxide/iron (GO/ $\mathrm{Fe}_{3} \mathrm{O}_{4}$ ) nanocomposites and DNA aptamer for quantifying E. coli O157:H7 in milk, avoiding tedious chemical modifications by combining aptamer self-assembly with GO and magnetic separation (Khang et al., 2016; Zhou et al., 2017). These $\mathrm{Fe}_{3} \mathrm{O}_{4} / \mathrm{GO}$ nanoparticles were also prepared by an intricate chemical coprecipitation method, and the sensing results could be inaccurate due to incomplete adsorption of $\mathrm{Fe}_{3} \mathrm{O}_{4} / \mathrm{GO}$ nanoparticles for unbound aptamer from solution because nanocomposites were added in the mixture after incubation of aptamer and target bacteria. The surface area of GO is not as large as MWCNT for a nanoparticle, which could adsorb fewer single-stranded DNA aptamers. Thus, the fabrication of carbon nanomaterials with magnetism must be created through a novel approach, and the accuracy of the aptasensor needs to be improved.

In the present study, we introduced a novel, simple, and safe synthetic method for magnetic carbon nanomaterials without irritating and corrosive reagents. Afterward, the CIP@MWCNT-based aptasensor was modified by noncovalent functionalization concatenation due to $\pi-\pi$ stacking of CIP@MWCNT nanocomplex with a dye-labeled aptamer targeting $E$. coli O157:H7. This caused fluorescent quenching and the restoration of fluorescence could be achieved when aptamer combined with E. coli O157:H7. The detailed construction of nanocomposite and multiple performance analysis of the aptasensor were evaluated to verify the effectiveness. The neo-aptasensor in this research provides a simple, rapid, and sensitive system for detecting pathogens for dairy safety.

\section{MATERIALS AND METHODS}

\section{Materials and Reagents}

Carboxylated MWCNT with an outer diameter $<8$ $\mathrm{nm}$ were purchased from Nanjing XFNANO Materials Tech Co. Ltd. (Nanjing, China). Carbonyl iron powder (CIP) was obtained from Jilin Zhuochuang New
Materials Co. Ltd. (Jilin, China). Ethanesulfonic acid and $N$-hydroxysuccinimide (NHS) were purchased from Shanghai Aladdin Biochemical Technology Co. Ltd. (Shanghai, China). Carbodiimide hydrochloride (EDC), anti-E. coli O157:H7 aptamer (Wu et al., 2012), conjugated fluorescein amidite (FAM), as shown below, were purchased from Sangon Biotech Co. Ltd. (Shanghai, China) and purified using HPLC. All other reagents used in this work were of analytical grade and solutions were prepared with ultrapure water.

The chosen aptamer could specifically bind to LPS of E. coli $\mathrm{O} 157: \mathrm{H} 7$ according to the report by Wu et al. (2012): 5'-FAM-CCGGACGCTTATGCCTTGCCA TCTACAGAGCAGGTGTGACGG-3' .

\section{Bacterial Strains}

Escherichia coli O157:H7 ATCC 25922 and other common pathogenic bacteria associated with dairy products and human health used in this research were cultured in Luria-Bertani broth under gentle shaking $(150 \mathrm{rpm})$ at $37^{\circ} \mathrm{C}$ and remained overnight. Information about these strains is shown in Table 1. Cell concentrations were determined by plating on Luria-Bertani agar and the cultivated bacteria were washed 3 times with an aseptic phosphate buffer solution (PBS, $0.1 \mathrm{M}$, pH $=7.4$ ).

\section{Fabrication and Characterization of the CIP@MWCNT Nanocomposites}

The carboxylated MWCNT $(1 \mathrm{mg} / \mathrm{mL})$ were activated and stabilized with EDC (40 $\mathrm{m} M, 100 \mu \mathrm{L})$ and NHS $(40 \mathrm{mM}, 100 \mu \mathrm{L})$ into a clean glass bottle that contained ethanesulfonic acid buffer $(0.1 \mathrm{M}, \mathrm{pH} 5.0)$ with shaking $(180 \mathrm{rpm})$ for $2 \mathrm{~h}$ at room temperature (RT). Afterward, CIP and MWCNT were blended at a mass ratio of $3: 1$, and the mixture was conducted by shaking (180 rpm) for $60 \mathrm{~min}$ at RT. The nanocomposites were magnetically separated and washed repeatedly with deionized water to remove excess reagents. The complex was dried for several hours and stored at RT following application. All above reagents used in this work were filtered through Millipore Express Membrane Filters (0.22 $\mu \mathrm{m}$, Merck Millipore Ltd., Billerica, MA). The zeta potential of nanomaterials was measured utilizing dynamic laser light scattering (Zetasizer Nano ZS90, Malvern Instruments Ltd., Malvern, UK). The surface functional groups from nanocomposites were probed by Fourier-transform infrared spectroscopy (FT-IR, Thermo Fisher, Waltham, MA). The morphology of nanomaterials was observed using transmission electron microscopy (H-7650, Hitachi High-Technologies Corporation, Tokyo, Japan). The element of nanocomplex was 
measured by energy dispersive x-ray analysis (EDAX, S-3400N, Hitachi High-Technologies Corporation).

\section{Preparation of the CIP@MWCNT-Based Aptasensor}

The CIP@MWCNT-based aptasensor was prepared according to Barbosa et al. (2019) with modifications. The CIP@MWCNT nanocomplex was dispersed in deionized water by an ultrasound bath (model KQ-500DE, 500 W, 40 kHz, Kunshan Ultrasonic Instrument Co. Ltd., Kunshan, Jiangsu, China) at a concentration of $1 \mathrm{mg} / \mathrm{mL}$ for $60 \mathrm{~min}$ at $20^{\circ} \mathrm{C}$. Then, the FAM-aptamer (100 n M, $100 \mu \mathrm{L})$ was mixed with dispersed CIP@ MWCNT complex, and the dispersion was treated in deionized water by sonication $(500 \mathrm{~W}, 40 \mathrm{kHz})$ for 60 min at $20^{\circ} \mathrm{C}$. The compounds were maintained overnight at $4^{\circ} \mathrm{C}$ to attain equilibrium after sonication. Subsequently, the nanocomplex was magnetically separated and washed with deionized water to remove excess aptamer. The washing steps were repeated 4 times and the efficiency of removal free aptamer was measured by a fluorescence microplate reader (model Infinite 200 PRO, Tecan, Grödig, Austria; $\lambda_{\text {excitation }}=492 \mathrm{~nm}$, $\left.\lambda_{\text {emission }}=532 \mathrm{~nm}\right)$. After washing, the CIP@MWCNT -based aptasensor pellet was resuspended in autoclaved deionized water.

\section{Principle of the Sensing Strategy}

Figure 1 illustrates the analytical process for E. coli O157:H7 on the CIP@MWCNT-based aptasensor. The magnetic nanocompounds based on CIP and MWCNT were formed by redox reaction. The CIP reacted with $\mathrm{H}^{+}$in acidic solution to form positively charged $\mathrm{Fe}^{2+}$ that adsorbed on the surface of nanoparticles. Under the attraction of the opposite charge, MWCNT with negative charge would bind with CIP particles, which were tightly adsorbed to form magnetic nanocomplexes. The chemical reaction formulas that took place are shown below. Then, the FAM-labeled aptamer wrapped randomly around the sidewalls of CIP@MWCNT due to $\pi-\pi$ stacking interactions, and the FAM molecules were in close proximity to the CIP@MWCNT, effectively quenching the fluorescence of the dye. In the presence of E. coli O157:H7, the aptamer left the sidewalls of CIP@ MWCNT, causing augmented fluorescence because the

Table 1. The specificity of CIP@MWCNT-aptasensor assay with selected strains ${ }^{1}$

\begin{tabular}{llll}
\hline No. & Strain & Source $^{2}$ & Results $^{3}$ \\
\hline 1 & Escherichia coli O157:H7 & ATCC 25922 & + \\
2 & Escherichia coli O157:H7 & CICC 21530 & + \\
3 & Escherichia coli O157:H7 & CECT 4783 & - \\
4 & Escherichia coli O111 & CMCC 44151 & - \\
5 & Escherichia coli & ATCC 43895 & - \\
6 & Escherichia coli & CMCC 44825 & - \\
7 & Cronobacter sakazakii & ATCC 29544 & - \\
8 & Cronobacter turicensis & DSM 18703 & - \\
9 & Cronobacter malonaticus & DSM 18702 & - \\
10 & Salmonella typhimurium & ATCC 14028 & - \\
11 & Salmonella typhimurium & ATCC 23564 & - \\
12 & Salmonella Dublin & CMCC 50042 & - \\
13 & Staphylococcus aureus & ATCC 13565 & - \\
14 & Staphylococcus aureus & CMCC 26075 & - \\
15 & Bacillus cereus & ATCC 14579 & - \\
16 & Bacillus subtilis & Laboratory & - \\
17 & Enterobacter aerogenes & ATCC 13048 & - \\
18 & Enterobacter cloacae & CMCC 45301 & - \\
19 & Enterobacter cloacae & CMCC 3503 & - \\
20 & Listeria monocytogenes & ATCC 19114 & - \\
21 & Listeria monocytogenes & ATCC 19116 & - \\
22 & Listeria welshimeri & ATCC 43550 & - \\
23 & Micrococcus luteus & Laboratory & - \\
24 & Vibrio parahaemolyticus & ATCC 33847 & - \\
25 & Enterococcus faecalis & Laboratory & \\
26 & Shigella flexneri & CICC 21678 & CMCC 51572 \\
27 & Shigella flexneri & &
\end{tabular}

${ }^{1} \mathrm{CIP}=$ carbonyl iron powder; MWCNT $=$ multiwalled carbon nanotubes.

${ }^{2}$ ATCC $=$ American Type Culture Collection (Manassas, VA); CMCC = China Medical Culture Collection (Beijing, China); CICC = China Center of Industrial Culture Collection (Beijing, China); DSM = DSMZDeutsche Sammlung von Mikroorganismen und Zellkulturen (Braunschweig, Germany); CECT = Colección Española de Cultivos Tipo (Valencia, Spain).

${ }^{3}+=$ positive results; $-=$ negative results. 

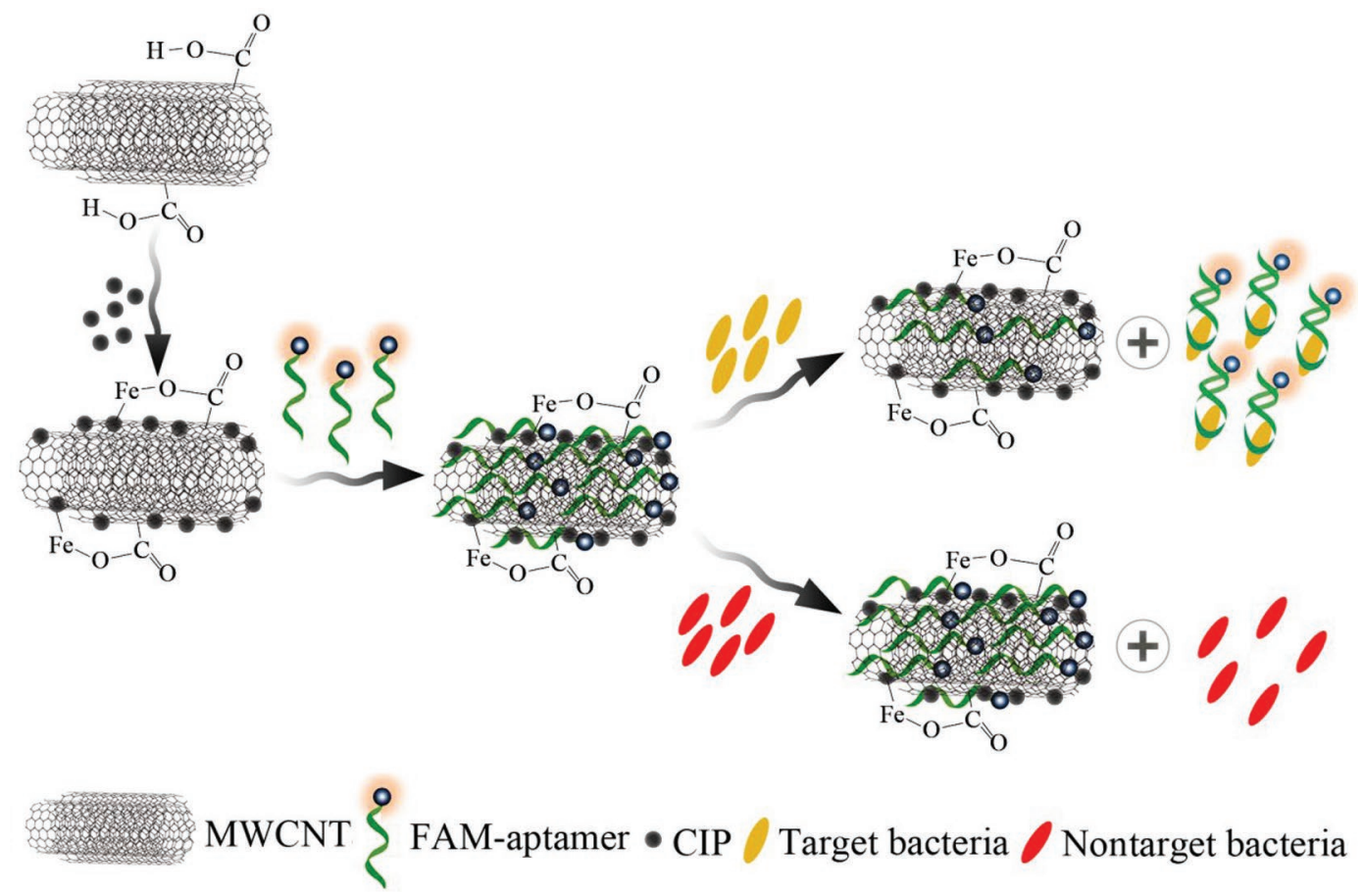

Figure 1. Schematic illustration of the CIP@MWCNT-based aptasensor for the detection of Escherichia coli O157:H7. CIP = carbonyl iron powder; MWCNT = multiwalled carbon nanotubes; FAM = conjugated fluorescein amidite.

aptamer had higher affinity and specificity against $E$. coli O157:H7 than CIP@MWCNT. Conversely, the fluorescence was turned off because the aptamer was still adsorbed onto the CIP@MWCNT.

$$
\begin{aligned}
& \text { CIP-Fe }+\mathrm{H}^{+} \rightarrow \text { CIP-Fe }{ }^{2+}+\mathrm{H}_{2}, \\
& \mathrm{MWCNT}-\mathrm{COOH} \rightarrow \mathrm{MWCNT}-\mathrm{COO}^{-}+\mathrm{H}^{+}, \\
& \text {CIP-Fe }{ }^{2+}+\text { MWCNT-COO }^{-} \\
& \rightarrow \text { MWCNT-COOFe-CIP. }
\end{aligned}
$$

\section{Implementation of the Protocol for Detection}

The system containing $500 \mu \mathrm{L}$ of $E$. coli $\mathrm{O} 157: \mathrm{H} 7$ and $50 \mu \mathrm{L}$ of CIP@MWCNT-based aptasensor was vortexed for $20 \mathrm{~s}$ in a black microcentrifuge tube. Then, the mixing solution was incubated for $10 \mathrm{~min}$ at $37^{\circ} \mathrm{C}$ in an incubator. Then $200 \mu \mathrm{L}$ of mixture was taken in a black 96-well polystyrene plate. The fluorescence intensity (FI) was tested by a microplate reader at $532 \mathrm{~nm}$ with an excitation wavelength of $492 \mathrm{~nm}$. The whole process applied black vessels and avoided light treatment to protect the fluorophore. Each test was repeated in triplicate. Line charts were recorded as digital documents for subsequent reset and analysis.

\section{Analytical Specificity, Sensitivity, Reproducibility, and Stability}

Each solution $(500 \mu \mathrm{L})$ containing target bacteria was vortexed with a CIP@MWCNT-based aptasensor $(50 \mu \mathrm{L})$ in microcentrifuge tubes. The FI was measured by fluorescence microplate reader $\left(\lambda_{\text {excitation }}=492 \mathrm{~nm}\right.$, $\left.\lambda_{\text {emission }}=532 \mathrm{~nm}\right)$ after the mixing solutions were incubated at $37^{\circ} \mathrm{C}$ for $10 \mathrm{~min}$. The specificity of the CIP@ MWCNT-based aptasensor was proven against a cluster of pathogenic bacteria (Table 1) considered to be hazardous in food under the same cell concentrations as target detection for E. coli O157:H7. The E. coli O157:H7 was 10-fold serially diluted to obtain different cell concentrations prepared in PBS buffer ranging from $10^{7}$ to $10^{1} \mathrm{cfu} / \mathrm{mL}$ and each dilution was used to analyze sensitivity. In the reproducibility analysis, 8 of the same batch and different batches of CIP@MWCNT -based aptasensor were randomly selected to test the intragroup differences and intergroup differences. Each sample $(500 \mu \mathrm{L})$ containing $E$. coli $\mathrm{O} 157: \mathrm{H} 7$ was vortexed with a CIP@MWCNT-based aptasensor $(50 \mu \mathrm{L})$ from different groups in microcentrifuge tubes. The bacterial concentration of $10^{7} \mathrm{cfu} / \mathrm{mL}$ was used for each test. After the mixing solutions were incubated for 10 min at $37^{\circ} \mathrm{C}$, the FI was measured by fluorescence microplate reader $\left(\lambda_{\text {excitation }}=492 \mathrm{~nm}, \lambda_{\text {emission }}=532\right.$ 
$\mathrm{nm})$. The relative standard deviation (RSD) values were calculated and compared with $5 \%$. In general, a good reproducibility is indicated by RSD values of less than 5\% (Sun et al., 2018). Furthermore, we evaluated the stability of this system for washing E. coli O157:H7 (estimated $10^{7} \mathrm{cfu} / \mathrm{mL}$ ) by utilizing the CIP@MWCNT -based aptasensor stored at $4^{\circ} \mathrm{C},-20^{\circ} \mathrm{C}$, and RT. Other conditions were maintained equally and the CIP@ MWCNT-based aptasensor was tested for $7 \mathrm{wk}$, and the interval of measurement was $1 \mathrm{wk}$. For all the above project analyses, a negative control (NC) was designed with PBS and the FI of the assay was tested at 532 $\mathrm{nm}$ with an excitation wavelength of $492 \mathrm{~nm}$. These indexes were evaluated using the CIP@MWCNT-based aptasensor in 3 replications. Line charts were recorded as digital documents for subsequent reset and analysis.

\section{Artificially Contaminated Milk with E. coli 0157:H7}

Commercially available UHT sterilized milk was used to verify the suitability of the sensing assay of the CIP@ MWCNT-based aptasensor. The UHT sterilized milk was purchased from supermarkets in Harbin, China, and verified by the conventional culturing method for the absence of $E$. coli O157:H7 (ISO, 2001). The UHT sterilized milk containing several different concentrations (e.g., $10^{0}$ to $10^{6} \mathrm{cfu} / \mathrm{mL}$ ) of E. coli $\mathrm{O} 157: \mathrm{H} 7$ was prepared in PBS $(0.1 M, \mathrm{pH}=7.4)$. The $\mathrm{NC}$ group was prepared with UHT sterilized milk, which had no artificial contamination of $E$. coli $\mathrm{O} 157: \mathrm{H} 7$. The artificially contaminated milk samples and NC group were simultaneously incubated by shaking $(180 \mathrm{rpm})$ for $1 \mathrm{~h}$ at $37^{\circ} \mathrm{C}$. Each sample $(500 \mu \mathrm{L})$ was vortexed with CIP@ MWCNT-based aptasensor $(50 \mu \mathrm{L})$ in microcentrifuge tubes. The FI was tested at $532 \mathrm{~nm}$ with an excitation wavelength of $492 \mathrm{~nm}$ after the mixture was incubated at $37^{\circ} \mathrm{C}$ for $10 \mathrm{~min}$. These procedures were conducted using the CIP@MWCNT-based aptasensor in 3 replications. Line charts were recorded as digital documents for subsequent reset and analysis.

\section{RESULTS}

\section{Characterization of the CIP@MWCNT Nanocomposite}

Figure 2A and B show pure MWCNT and CIP@ MWCNT nanocomposite in $\mathrm{H}_{2} \mathrm{O}$. The CIP@MWCNT nanocomposite was more easily aggregated, which could be relative with the result of transform in charge after the redox reaction. As shown in Figure 2C and D, CIP@MWCNT were attracted by the magnetic stand in aqueous solution, but MWCNT were not. Furthermore, 3 kinds of characterization from different aspects were performed to verify the formation of nanocomposite. Zeta potential and FT-IR spectra are common characterizations of surface charge and functional groups from nanoparticles. As shown in Figure 3A, the zeta potential of MWCNT and CIP was -21.7 and 3.4 $\mathrm{mV}$, respectively, whereas the zeta potential of CIP@ MWCNT was $-1.4 \mathrm{mv}$. The reduction of the negative charge might be redox reaction in liquor, and decrease of the same charge could lead to more aggregation. Figure $3 \mathrm{~B}$ shows the FT-IR spectra of pristine MWCNT, CIP, and CIP@MWCNT. The absorption peaks of O-H and $\mathrm{C}=\mathrm{O}$ appeared at 3,425 and $1,682 \mathrm{~cm}^{-1}$, respectively, which was due to the carboxyl functional groups on MWCNT and CIP@MWCNT. The new peak at 610 $\mathrm{cm}^{-1}$ was ascribed to the $\mathrm{Fe}-\mathrm{O}$ bond. The consumption of $\mathrm{O}-\mathrm{H}$ and generation of $\mathrm{Fe}-\mathrm{O}$ about signal intensity were attributed to the formation of CIP@MWCNT complexes. In addition, transmission electron microscopy images in Figure 4 revealed that CIP@MWCNT was synthesized successfully. In the present experiment, morphology, texture, and diameter were well preserved comparing MWCNT with CIP@MWCNT in Figure 4A and $\mathrm{B}$. The pristine MWCNT appeared to be reuniting, twisting, and having a large length distribution; nevertheless, some of the MWCNT were slightly curled and exfoliated after redox reaction. Furthermore, the dispersity of CIP@MWCNT was enhanced after the composite reaction. According to the results in Figure $4 \mathrm{C}$, certain CIP were distinctly observed on the outer walls of the MWCNT. The EDAX result showed that the amount of Fe doped into the MWCNT was about $93.25 \mathrm{wt} \%$ (Figure 4D). This was verified to determine the CIP in the nanocomposite materials. All of these results implied that the CIP and MWCNT were successfully connected, even in tiny diameters $(<8 \mathrm{~nm})$ of MWCNT that were purchased as minimum sizes from Nanjing XFNANO Materials Tech Co. Ltd.

\section{Determination and Evaluation of the CIP@MWCNT -Based Aptasensor}

The washing times of the preparation process needed to be determined to achieve higher accuracy. Figure 5 illustrates the consequence of the wash steps to remove excess aptamer by fluorescent detection. A gradual decline in fluorescence signal values was examined and the FI changes of supernatant were no longer monitored after 4 successive washes, which were consistent with the decreased amount of aptamer in these residues. Thus, the 4 washing times were handled following subsequent operation after the anti-E. coli O157:H7 aptamer and CIP@MWCNT complex were connected.

To verify the advantage of the CIP@MWCNT-based aptasensor, the specificity, sensitivity, reproducibility, 

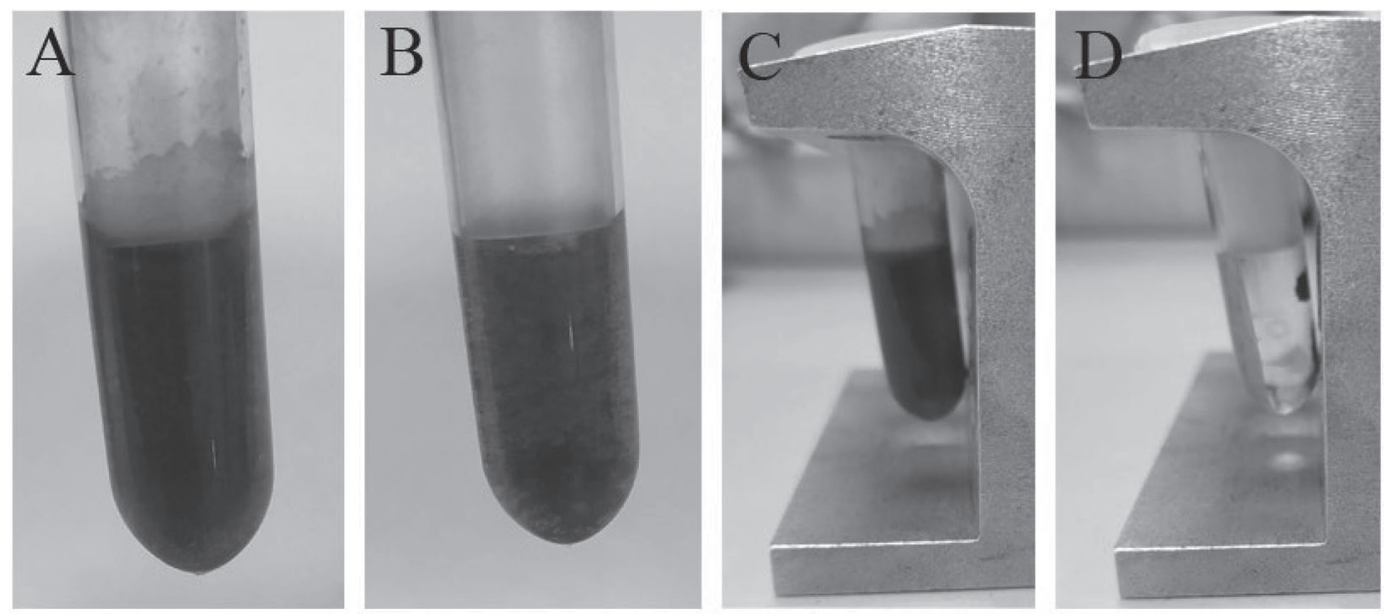

Figure 2. (A) Multiwalled carbon nanotubes (MWCNT) and (B) CIP@MWCNT nanomaterials in $\mathrm{H}_{2} \mathrm{O}$. (C) MWCNT and (D) CIP@ MWCNT nanomaterials in $\mathrm{H}_{2} \mathrm{O}$ using a magnetic stand. $\mathrm{CIP}=$ carbonyl iron powder.

and stability analyses were examined. From the results of Table 1, the CIP@MWCNT-based aptasensor had excellent specificity toward E. coli O157:H7 over the other pathogenic bacteria. The analytical sensitivity of the CIP@MWCNT-based aptasensor assay was determined by diluting cell concentrations of $E$. coli $\mathrm{O} 157: \mathrm{H} 7$ from $10^{7}$ to $10^{1} \mathrm{cfu} / \mathrm{mL}$. As shown in Figure 6 , the resulting calibration curve hinted that the FI was proportional to the concentration of the target in the dynamic range from $10^{3}$ to $10^{7} \mathrm{cfu} / \mathrm{mL}$ (Figure $6 \mathrm{~A}$ ) and a good linear response to E. coli $\mathrm{O} 157: \mathrm{H} 7$ was acquired in the range from $10^{4}$ to $10^{7} \mathrm{cfu} / \mathrm{mL}$ (Figure $6 \mathrm{~B}$ ). The linear regression equation is $\mathrm{FI}=342.6 \log [E$. coli $\mathrm{O} 157: \mathrm{H} 7$ $(\mathrm{cfu} / \mathrm{mL})]-1,200.8$ with a correlation coefficient of 0.9902. Limit of detection of the CIP@MWCNT-based aptasensor was as low as $7.15 \times 10^{3} \mathrm{cfu} / \mathrm{mL}$ in pure culture. It was calculated as background $+3 \mathrm{~s}$, which is explained as the concentration of sample generating signal intensity in accordance with the average of back- ground plus 3 times its standard deviation (Chen et al., 2016; Khang et al., 2016; Hassan et al., 2019; Huang et al., 2019). Accuracy is another pivotal factor of the quantitative assays (Sun et al., 2017), and reproducibility was executed with CIP@MWCNT-based aptasensor by comparing identical and different batches $(8$ parallel for each group). We found that the RSD values of intragroup and intergroup were $3.57 \%$ and $4.43 \%$, respectively (Figure 6C), and the measured values of latter were more dispersed. Hence the RSD values of both showed that the CIP@MWCNT-based aptasensor had good reproducibility $(<5 \%)$. Meanwhile, the stability of the nanomaterial-based biosensor, known as a significant element (Yao et al., 2016), was also considered in this work. From the results shown in Figure 6D, the fluorescent signals of the CIP@MWCNT -based aptasensor had no significant decline over $4 \mathrm{wk}$, and were always above the initial value of $94.1 \%$. Of note, the fluorescent signal started to decrease after
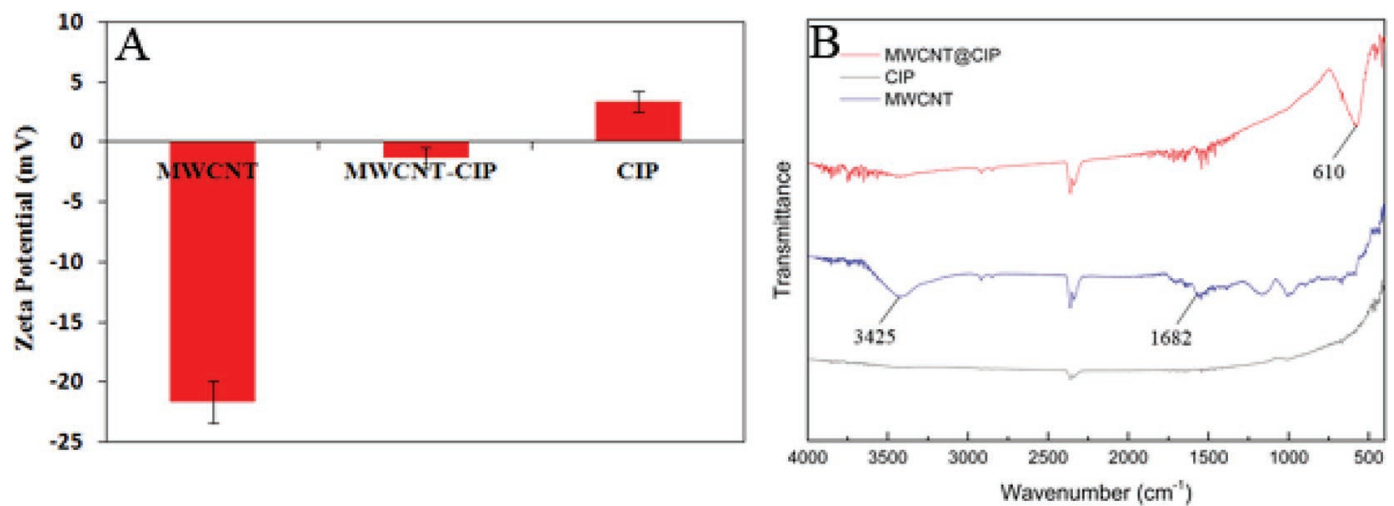

Figure 3. (A) Zeta potential and (B) Fourier-transform infrared spectroscopy spectra of multiwalled carbon nanotubes (MWCNT), carbonyl iron powder (CIP), and CIP@MWCNT. Error bars $=$ mean $\pm \mathrm{SD}(\mathrm{n}=3)$. 

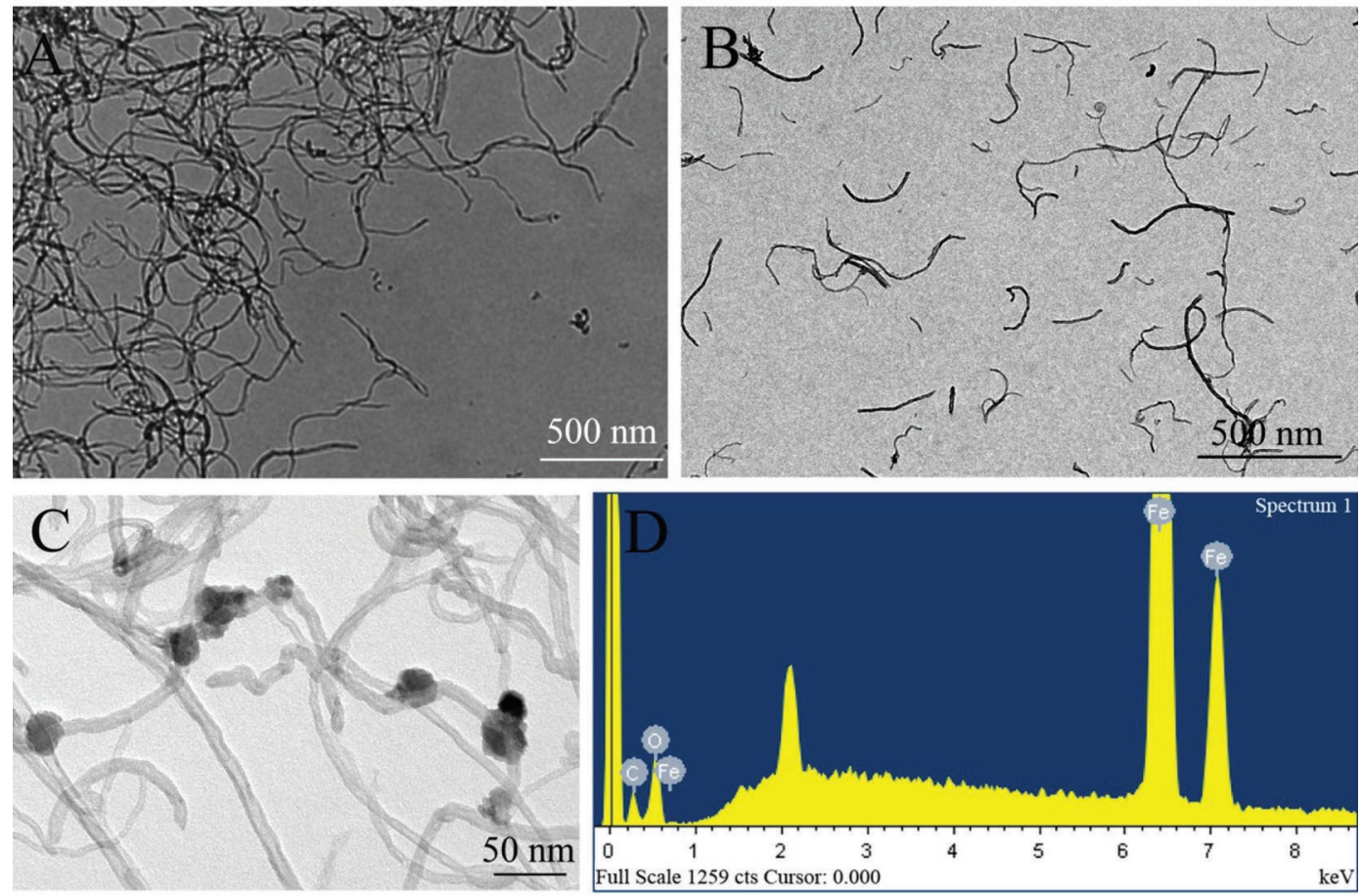

Figure 4. Transmission electron microscopy images of (A) pristine multiwalled carbon nanotubes (MWCNT) and (B and C) CIP@MWCNT. (D) Energy dispersive x-ray analysis results of CIP@MWCNT. CIP = carbonyl iron powder.

4 wk. Although the fluorescent values were still above $91.2 \%$ of the initial value at $4^{\circ} \mathrm{C}$ and $-20^{\circ} \mathrm{C}$, the fluorescent intensity had decreased to $79.9 \%$ stored at RT. These implied that low temperature could maintain the properties of the fluorophore. Thus, the CIP@MWCNT -based aptasensor had good fluorescence stability at 3 usual temperatures for almost a month.

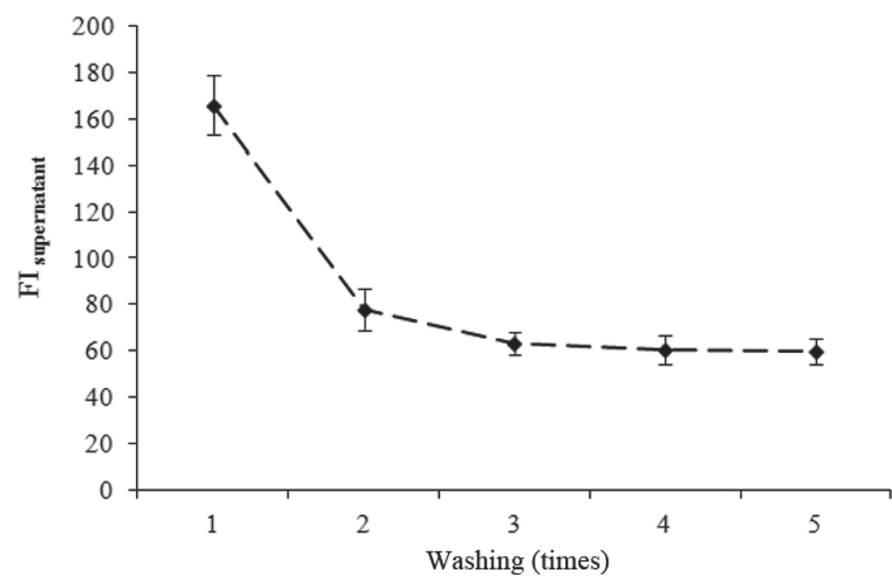

Figure 5. Fluorescence values for residues in the washing. FI = fluorescence intensity. Error bars $=$ mean $\pm \mathrm{SD}(\mathrm{n}=3)$.

\section{Quantification of CIP@MWCNT-Based Aptasensor in Milk}

We further explored the performance of the CIP@ MWCNT-based aptasensor with spiked E. coli O157:H7 in UHT sterilized milk. The aptasensor had a higher level for directly detecting concentration of $E$. coli O157:H7 in milk $\left(10^{4} \mathrm{cfu} / \mathrm{mL}\right)$ without incubation than pure culture $\left(10^{3} \mathrm{cfu} / \mathrm{mL}\right)$, which was attributed to the matrix interference of milk. Similar interference of milk on other technologies for detecting E. coli $\mathrm{O} 157: \mathrm{H} 7$ was previously reported (Aydin et al., 2014; Khang et al., 2016; Luo et al., 2017; Han et al., 2018) and incubation was required. In Figure 7, the results of the calibration curve hinted that the FI was proportional to the concentration of target in the dynamic range from $10^{2}$ to $10^{6} \mathrm{cfu} / \mathrm{mL}$ (Figure $7 \mathrm{~A}$ ) after incubation at $37^{\circ} \mathrm{C}$ for $1 \mathrm{~h}$. A good linear response to E. coli $\mathrm{O} 157: \mathrm{H} 7$ was acquired in the range from $10^{3}$ to $10^{6} \mathrm{cfu} / \mathrm{mL}$ (Figure 7B). The linear regression equation is $\mathrm{FI}=421.9 \mathrm{log}$ [E. coli O157:H7 (cfu/mL)] - 995.13 with a correlation coefficient of 0.9916 . Limit of detection of the CIP@ MWCNT-based aptasensor was as low as $3.15 \times 10^{2}$ $\mathrm{cfu} / \mathrm{mL}$ in UHT sterilized milk after $1 \mathrm{~h}$ of pre-incubation. It was calculated as background $+3 \mathrm{~s}$, which is explained as the concentration of sample generating signal intensity in accordance with the average of back- 

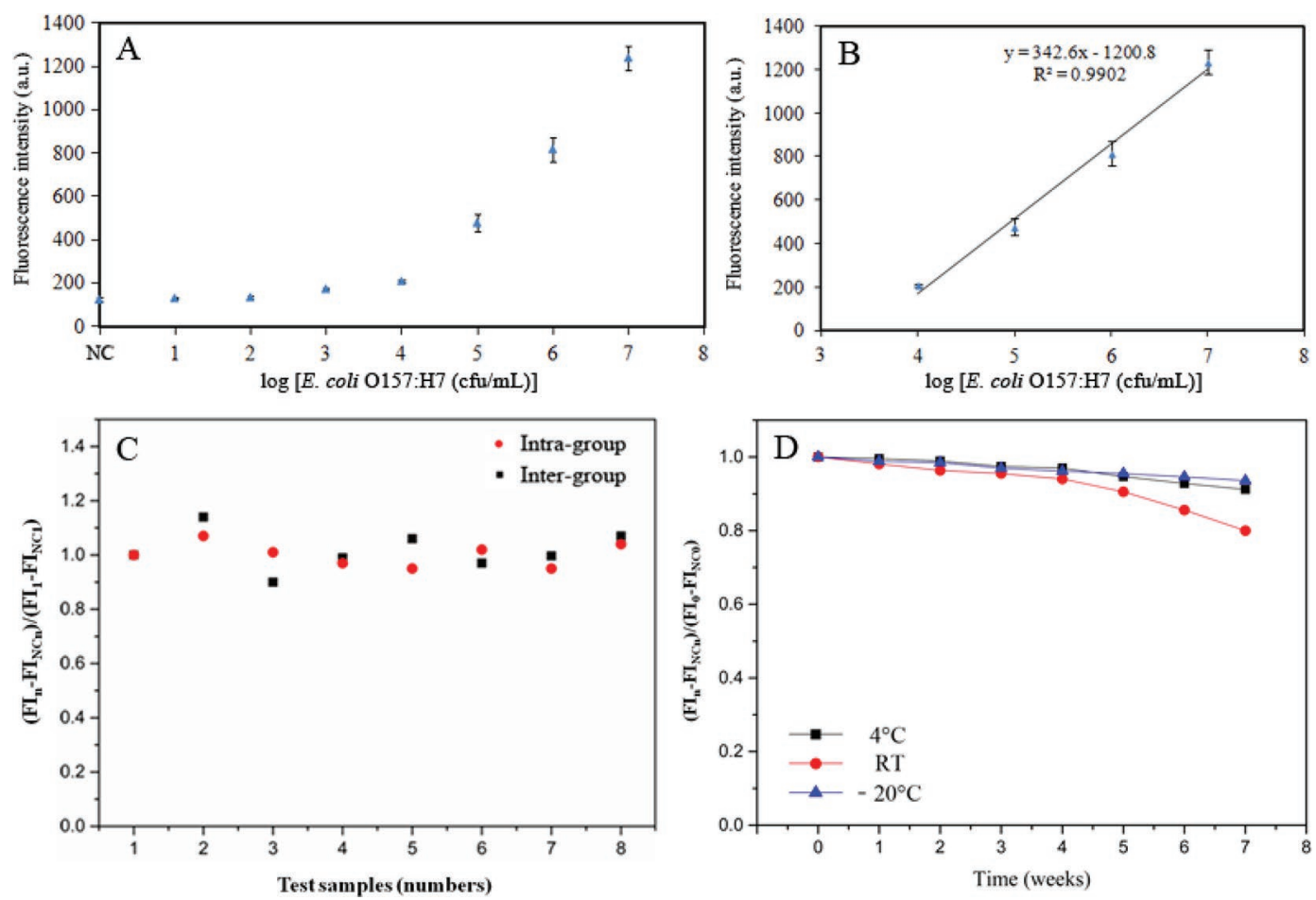

Figure 6. (A and B) Sensitivity, (C) reproducibility, and (D) stability of the CIP@MWCNT-based aptasensor for Escherichia coli O157:H7 in pure culture. a.u. = arbitrary units; $\mathrm{NC}=$ negative control; $\mathrm{FI}_{\mathrm{NC}}=$ fluorescence intensity $(\mathrm{FI})$ value of negative control $\mathrm{FI}=\mathrm{FI}$ value of the nth sample, $\mathrm{n}=1,2,3,4,5,6,7,8 ; \mathrm{FI}_{1}=\mathrm{FI}$ value of the first sample; $\mathrm{RT}=$ room temperature. $\mathrm{CIP}=$ carbonyl iron powder; $\mathrm{MWCNT}=$ multiwalled carbon nanotubes. Error bars $=$ mean $\pm \mathrm{SD}(\mathrm{n}=3)$.

ground plus 3 times its standard deviation (Chen et al., 2016; Khang et al., 2016; Hassan et al., 2019; Huang et al., 2019). Thus, the CIP@MWCNT-based aptasensor had a capacity of rapidly binding $E$. coli $\mathrm{O} 157: \mathrm{H} 7$ in an authentic sample with excellent sensitivity.

\section{DISCUSSION}

The utilization of carbon materials with nanoscale for preparation of biosensors has had increasing atten- tion following the discovery of carbon nanotubes by Sumio Ijima in 1991 (Sadik et al., 2009). Some studies reported that solvothermal and deposition in situ synthesis of magnetic carbon nanomaterials were widely used, but corrosive and irritating agents were needed in these methods, which could be harmful to the body in case of maloperation. In the present experiment, we synthesized the CIP@MWCNT nanocomposite by a safe and effective one-step method for the first time. The carboxylated MWCNT were activated with EDC
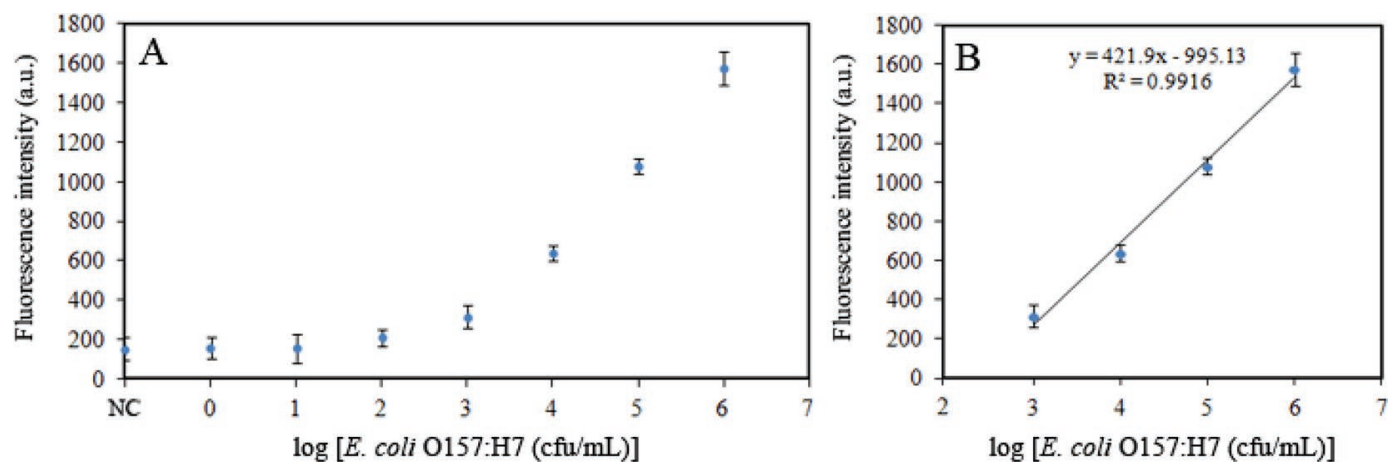

Figure 7. Sensitivity of the CIP@MWCNT-based aptasensor for Escherichia coli O157:H7 in milk. NC = negative control; CIP = carbonyl iron powder; MWCNT $=$ multiwalled carbon nanotubes. Error bars $=$ mean $\pm \mathrm{SD}(\mathrm{n}=3)$. 
and stabilized with NHS, which conquered agminate restriction of MWCNT and improved the solubility in aqueous medium (Nabiabad et al., 2018). The union of EDC/NHS would also serve as a conjugate to facilitate integration of 2 kinds of nanomaterials (Chavan et al., 2019). Then, the compound was obtained by a one-step method based on redox reaction. This would be a promising approach for the synthesis of magnetic carbon nanocomposites due to its rapidity and safety.

Otherwise, the aptasensor was formed from the aptamer carried by CIP@MWCNT, rather than the free aptamer absorbed by nanocomposite after incubation of bacteria and aptamer. This ensured that the entire aptamer of the system came from the CIP@MWCNT -based aptasensor, which was a more accurate detecting strategy that differed from previous assays (Wang and $\mathrm{Si}, 2013)$. In preparation, the application of ultrasound helped to enhance the dissolution and diffusion capacities of the CIP@MWCNT nanocomposite, which facilitated higher reaction rates, more uniform polymer formation, and higher yields (Ncibi and Sillanpaa, 2015). The CIP@MWCNT-based aptasensor was effective for detection of E. coli O157:H7 via thoughtful evaluation of specificity, sensitivity, reproducibility, and stability. Meanwhile, the aptasensor was valid and sensitive for detection of E. coli O157:H7 in milk. The detection limit of the present report was acceptable, and was also compared with other detection methods toward E. coli O157:H7 (Table 2). The chemiluminescence- $\mathrm{Fe}_{3} \mathrm{O}_{4} / \mathrm{GO}$ (Khang et al., 2016) and electrochemical (Luo et al., 2012; Xu et al., 2016) detected E. coli O157:H7 of 4.5 $\times 10^{3}, 3.05 \times 10^{3}$, and $2.05 \times 10^{3} \mathrm{cfu} / \mathrm{mL}$ in skim milk, milk, and ground beef, respectively. Additionally, some research reported E. coli O157:H7 of $1 \times 10^{3}$ and 1.46 $\times 10^{3} \mathrm{cfu} / \mathrm{mL}$ in meat and PBS solution using a fiber optic biosensor (Ohk and Bhunia, 2013) and quartz crystal microbalance (Yu et al., 2018). The sensitivity of the various fluorometric assays for E. coli O157:H7 was usually $10^{2} \mathrm{cfu} / \mathrm{mL}$ in different food samples (Chen et al., 2016; Li et al., 2018; Hassan et al., 2019; Huang et al., 2019; Zhang et al., 2019), as well as this work. In terms of detecting assays with nanotechnology, the CIP@MWCNT-based aptasensor has several advantages: (1) CIP@MWCNT nanocomposite can be obtained without irritating and corrosive reagents, thus avoiding dangerous and intricate procedures; (2) CIP@MWCNT shows a certain degree of stabilization to single-stranded aptamer by $\pi-\pi$ stacking, which can react satisfactorily at RT for up to a month; and (3) the CIP@MWCNT-based aptasensor can tolerate complex dairy matrices and detect pathogens with sensitivity. These distinguishing features showed that this assay could be a prospective way of inspecting pathogens in the dairy industry.

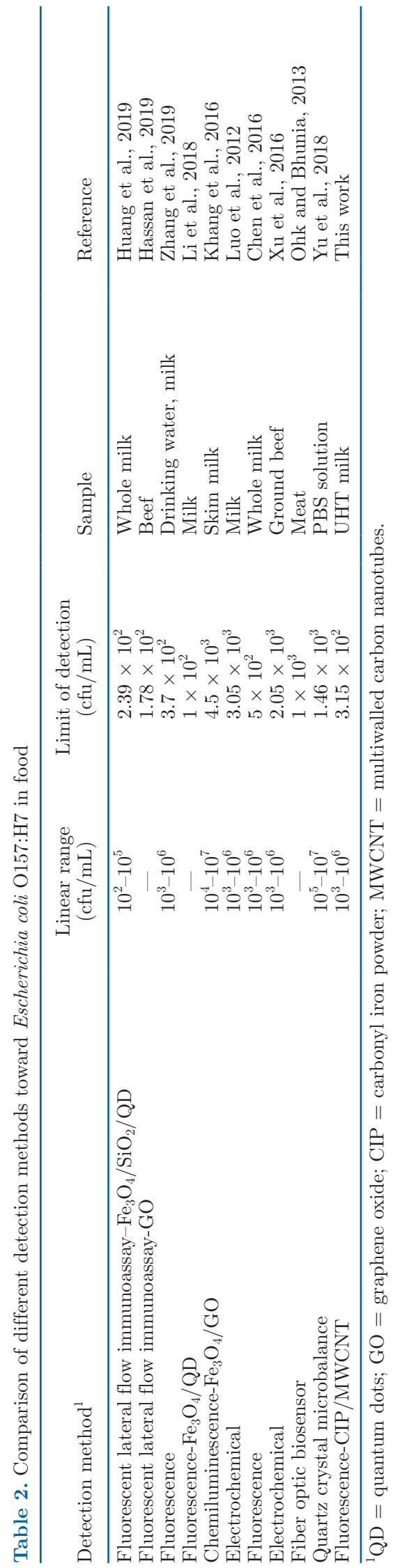




\section{CONCLUSIONS}

In summary, our newly developed CIP@MWCNT -based aptasensor with fluorescence detection could be successfully applied for sensitive diagnosis of $E$. coli O157:H7 in a contaminated sample matrix. Four kinds of characterization of surface charge, functional groups, and morphology revealed the successful synthesis of nanocomposites through CIP and MWCNT. Under the prepared system of aptasensor, E. coli O157:H7 could be detected at a concentration as low as $7.15 \times 10^{3}$ $\mathrm{cfu} / \mathrm{mL}$ in pure culture. The detection limit of $E$. coli O157:H7 was $3.15 \times 10^{2} \mathrm{cfu} / \mathrm{mL}$ in contaminated milk after $1 \mathrm{~h}$ of pre-incubation. In addition, this new type of magnetic nanocomposite modified aptasensor is reliable and stable with good targeting ability according to the performance analyses. All statistical information verified that the CIP@MWCNT-based aptasensor has potential for rapid, sensitive, and reliable detection of E. coli $\mathrm{O} 157: \mathrm{H} 7$ in a practical sample. In the future, this type of developed aptasensor will be used to simultaneously monitor multiple types of foodborne pathogens in real-world samples.

\section{ACKNOWLEDGMENTS}

This work was supported by the National Key Research and Development Program of China (No. 2018YFE0120500). The authors declare that they do not have any commercial or associative interest that represents a conflict of interest in connection with the work submitted.

\section{REFERENCES}

Aydin, M., G. P. D. Herzig, K. C. Jeong, S. Dunigan, P. Shah, and S. Ahn. 2014. Rapid and sensitive detection of Escherichia coli O157: $\mathrm{H} 7$ in milk and ground beef using magnetic bead-based immunoassay coupled with tyramide signal amplification. J. Food Prot. 77:100-105. https://doi.org/10.4315/0362-028X.JFP-13-274.

Barbosa, M. B., E. M. D. Martins, T. F. Teixeira, R. D. E. Carvalho, J. P. Coelho, R. R. Resende, E. F. Oliveira, A. P. Santos, A. S. R. de Andrade, and C. A. Furtado. 2019. A carefully designed nanoplatform based on multi walled carbon nanotube wrapped with aptamers. Colloids Surf. B Biointerfaces 175:175-183. https://doi .org/10.1016/j.colsurfb.2018.11.064.

Chavan, S., D. Kim, J. Hwang, Y. Choi, J. W. Hong, J. Kim, M. H. Lee, M. P. Hwang, and J. Choi. 2019. Enhanced detection of infectious pancreatic necrosis virus via lateral flow chip and fluorometric biosensors based on self-assembled protein nanoprobes. ACS Sens. 4:2937-2944. https://doi.org/10.1021/acssensors.9b01362.

Chen, R., X. L. Huang, J. Li, S. Shan, W. H. Lai, and Y. H. Xiong. 2016. A novel fluorescence immunoassay for the sensitive detection of Escherichia coli O157:H7 in milk based on catalase-mediated fluorescence quenching of CdTe quantum dots. Anal. Chim. Acta 947:50-57. https://doi.org/10.1016/j.aca.2016.10.017.

Demirkol, D. O., and S. Timur. 2016. A sandwich-type assay based on quantum dot/aptamer bioconjugates for analysis of E. coli $\mathrm{O} 157: \mathrm{H} 7$ in microtiter plate format. Int. J. Polym. Mater. Polym. Biomater. 65:85-90. https://doi.org/10.1080/00914037.2015.1074906.
Deng, J. H., X. H. Wen, and Q. N. Wang. 2012. Solvothermal in situ synthesis of $\mathrm{Fe}_{3} \mathrm{O}_{4}$-multi-walled carbon nanotubes with enhanced heterogeneous Fenton-like activity. Mater. Res. Bull. 47:33693376. https://doi.org/10.1016/j.materresbull.2012.07.021.

Gao, J. N., X. Z. Ran, C. M. Shi, H. M. Cheng, T. M. Cheng, and Y. P. Su. 2013. One-step solvothermal synthesis of highly watersoluble, negatively charged superpara magnetic $\mathrm{Fe}_{3} \mathrm{O}_{4}$ colloidal nanocrystal clusters. Nanoscale 5:7026-7033. https://doi.org/10 .1039/c3nr00931a.

Han, J. J., L. Zhang, L. M. Hu, K. Y. Xing, X. F. Lu, Y. J. Huang, J. W. Zhang, W. H. Lai, and T. Chen. 2018. Nanozyme-based lateral flow assay for the sensitive detection of Escherichia coli O157: H7 in milk. J. Dairy Sci. 101:5770-5779. https://doi.org/10.3168/jds .2018-14429.

Hassan, A. H. A., J. F. Bergua, E. Morales-Narvaez, and A. Mekoci. 2019. Validity of a single antibody-based lateral flow immunoassay depending on graphene oxide for highly sensitive determination of $E$. coli $\mathrm{O} 157: \mathrm{H} 7$ in minced beef and river water. Food Chem. 297:124965. https://doi.org/10.1016/j.foodchem.2019.124965.

Huang, Z., J. Peng, J. J. Han, G. G. Zhang, Y. J. Huang, M. L. Duan, D. F. Liu, Y. H. Xiong, S. Q. Xia, and W. H. Lai. 2019. A novel method based on fluorescent magnetic nanobeads for rapid detection of Escherichia coli O157:H7. Food Chem. 276:333-341. https: //doi.org/10.1016/j.foodchem.2018.09.164.

ISO. 2001. ISO 16654:2001. Microbiology of food and animal feeding stuffs - Horizontal method for the detection of Escherichia coli O157. https://www.iso.org/standard/29821.html.

Jamrozik, A., J. Przewoznik, M. Mazurkiewicz, A. Malolepszy, L. Stobinski, G. Trykowski, I. Habina, K. Matlak, J. Korecki, C. Kapusta, M. Sitarz, and K. Burda. 2014. Influence of iron contaminations on local and bulk magnetic properties of nonfunctionalized and functionalized multi-wall carbon nanotubes. Phys. Status Solidi., A Appl. Mater. Sci. 211:661-669. https://doi.org/10.1002/ pssa.201330150.

Khang, J., D. Kim, K. W. Chung, and J. H. Lee. 2016. Chemiluminescent aptasensor capable of rapidly quantifying Escherichia coli O157:H7. Talanta 147:177-183. https://doi.org/10.1016/j.talanta .2015.09.055.

Konni, M., A. S. Dadhich, and S. B. Mukkamala. 2017. Impact of surface modifications on hydrogen uptake by Fe@f-MWCNT and $\mathrm{Cu} @ f-M W C N T$ at non-cryogenic temperatures. Int. J. Hydrogen Energy 42:953-959. https://doi.org/10.1016/j.ijhydene.2016.09 .085 .

Krishna, V. D., K. Wu, D. Q. Su, M. C. J. Cheeran, J. P. Wang, and A. Perez. 2018. Nanotechnology: Review of concepts and potential application of sensing platforms in food safety. Food Microbiol. 75:47-54. https://doi.org/10.1016/j.fm.2018.01.025.

Li, L. Y., Q. J. Li, Z. Y. Liao, Y. Sun, Q. S. Cheng, Y. Song, E. Q. Song, and W. H. Tan. 2018. Magnetism-resolved separation and fluorescence quantification for near-simultaneous detection of multiple pathogens. Anal. Chem. 90:9621-9628. https://doi.org/ 10.1021/acs.analchem.8b02572.

Liu, L. F., D. Y. Li, C. L. Li, R. Ji, and X. F. Tian. 2018. Metal nanoparticles by doping carbon nanotubes improved the sorption of perfluorooctanoic acid. J. Hazard. Mater. 351:206-214. https:// doi.org/10.1016/j.jhazmat.2018.03.001.

Liu, X. L., R. Ma, X. X. Wang, Y. Ma, Y. P. Yang, L. Zhuang, S. Zhang, R. Jehan, J. R. Chen, and X. K. Wang. 2019. Graphene oxide-based materials for efficient removal of heavy metal ions from aqueous solution: A review. Environ. Pollut. 252:62-73. https:// doi.org/10.1016/j.envpol.2019.05.050.

Luo, C. H., Y. N. Lei, L. Yan, T. X. Yu, Q. Li, D. C. Zhang, S. J. Ding, and H. X. Ju. 2012. A rapid and sensitive aptamer-based electrochemical biosensor for direct detection of Escherichia coli O111. Electroanalysis 24:1186-1191. https://doi.org/10.1002/elan 201100700 .

Luo, K., L. M. Hu, Q. Guo, C. H. Wu, S. S. Wu, D. F. Liu, Y. H. Xiong, and W. H. Lai. 2017. Comparison of 4 label-based immunochromatographic assays for the detection of Escherichia coli O157: H7 in milk. J. Dairy Sci. 100:5176-5187. https://doi.org/10.3168/ jds.2017-12554. 
Nabiabad, H. S., K. Piri, and M. Amini. 2018. Expression of active chimeric-tissue plasminogen activator in tobacco hairy roots, identification of a DNA aptamer and purification by aptamer functionalized-MWCNT chromatography. Protein Expr. Purif. 152:137-145. https://doi.org/10.1016/j.pep.2016.02.004.

Ncibi, M. C., and M. Sillanpaa. 2015. Optimized removal of antibiotic drugs from aqueous solutions using single, double and multiwalled carbon nanotubes. J. Hazard. Mater. 298:102-110. https://doi .org/10.1016/j.jhazmat.2015.05.025.

Ohk, S. H., and A. K. Bhunia. 2013. Multiplex fiber optic biosensor for detection of Listeria monocytogenes, Escherichia coli O157:H7 and Salmonella enterica from ready-to-eat meat samples. Food Microbiol. 33:166-171. https://doi.org/10.1016/j.fm.2012.09.013.

Pourfadakari, S., N. Yousefi, and A. H. Mahvi. 2016. Removal of reactive red 198 from aqueous solution by combined method multiwalled carbon nanotubes and zero-valent iron: Equilibrium, kinetics, and thermodynamic. Chin. J. Chem. Eng. 24:1448-1455. https: //doi.org/10.1016/j.cjche.2016.04.027.

Qiu, W. W., H. Xu, S. Takalkar, A. S. Gurung, B. Liu, Y. F. Zheng, Z. B. Guo, M. Baloda, K. Baryeh, and G. D. Liu. 2015. Carbon nanotube-based lateral flow biosensor for sensitive and rapid detection of DNA sequence. Biosens. Bioelectron. 64:367-372. https: //doi.org/10.1016/j.bios.2014.09.028

Sadik, O. A., A. O. Aluoch, and A. L. Zhou. 2009. Status of biomolecular recognition using electrochemical techniques. Biosens. Bioelectron. 24:2749-2765. https://doi.org/10.1016/j.bios.2008.10 .003 .

Sun, L. H., Y. J. Jiang, R. L. Pan, M. Y. Li, R. Wang, S. H. Chen, S. Q. Fu, and C. X. Man. 2018. A novel, simple and low-cost paperbased analytical device for colorimetric detection of Cronobacter spp. Anal. Chim. Acta 1036:80-88. https://doi.org/10.1016/j.aca .2018.05.061

Sun, W. J., X. L. Hu, J. Liu, Y. R. Zhang, J. Z. Lu, and L. B. Zeng. 2017. A novel multi-walled carbon nanotube-based antibody conjugate for quantitative and semi-quantitative lateral flow assays. Biosci. Biotechnol. Biochem. 81:1874-1882. https://doi.org/10 .1080/09168451.2017.1365590.

Sun, X., J. P. He, G. X. Li, J. Tang, T. Wang, Y. X. Guo, and H. R. Xue. 2013. Laminated magnetic graphene with enhanced electromagnetic wave absorption properties. J. Mater. Chem. 1:765-777. https://doi.org/10.1039/c2tc00159d.

Wang, C. Q., J. Qian, K. Wang, K. Wang, Q. Liu, X. Y. Dong, C. K. Wang, and X. Y. Huang. 2015. Magnetic-fluorescent-targeting multifunctional aptasensor for highly sensitive and one-step rapid detection of ochratoxin A. Biosens. Bioelectron. 68:783-790. https: //doi.org/10.1016/j.bios.2015.02.008.

Wang, L. H., H. T. Guan, J. D. Hu, Q. Huang, C. J. Dong, W. Qian, and Y. D. Wang. 2019. Jute-based porous biomass carbon composited by $\mathrm{Fe}_{3} \mathrm{O}_{4}$ nanoparticles as an excellent microwave absorber. J.
Alloys Compd. 803:1119-1126. https://doi.org/10.1016/j.jallcom .2019.06.351.

Wang, S. E., and S. H. Si. 2013. Aptamer biosensing platform based on carbon nanotube long-range energy transfer for sensitive, selective and multicolor fluorescent heavy metal ion analysis. Anal. Methods 5:2947-2953. https://doi.org/10.1039/c3ay40360b.

Wu, W., S. M. Zhao, Y. P. Mao, Z. Y. Fang, X. W. Lu, and L. W. Zeng. 2015. A sensitive lateral flow biosensor for Escherichia coli O157:H7 detection based on aptamer mediated strand displacement amplification. Anal. Chim. Acta 861:62-68. https://doi.org/ 10.1016/j.aca.2014.12.041.

Wu, W. H., J. Z. Zhang, M. Q. Zheng, Y. H. Zhong, J. Yang, Y. H. Zhao, W. P. Wu, W. Ye, J. Wen, Q. Wang, and J. X. Lu. 2012 An aptamer-based biosensor for colorimetric detection of Escherichia coli O157:H7. PLoS One 7:e48999. https://doi.org/10.1371/ journal.pone.0048999.

Xu, M., R. H. Wang, and Y. B. Li. 2016. Rapid detection of Escherichia coli O157:H7 and Salmonella typhimurium in foods using an electrochemical immunosensor based on screen-printed interdigitated microelectrode and immunomagnetic separation. Talanta 148:200-208. https://doi.org/10.1016/j.talanta.2015.10.082.

Yao, L., J. Teng, M. Y. Zhu, L. Zheng, Y. H. Zhong, G. D. Liu, F. Xue, and W. Chen. 2016. MWCNT based high sensitive lateral flow strip biosensor for rapid determination of aqueous mercury ions. Biosens. Bioelectron. 85:331-336. https://doi.org/10.1016/j .bios.2016.05.031

Yu, X. F. F. Chen, R. H. Wang, and Y. B. Li. 2018. Whole bacterium SELEX of DNA aptamers for rapid detection of E.coli O157:H7 using a QCM sensor. J. Biotechnol. 266:39-49. https://doi.org/10 .1016/j.jbiotec.2017.12.011.

Zhang, Y., L. Q. Zhu, P. G. He, F. T. Zi, X. Z. Hu, and Q. J. Wang. 2019. Sensitive assay of Escherichia coli in food samples by microchip capillary electrophoresis based on specific aptamer binding strategy. Talanta 197:284-290. https://doi.org/10.1016/j.talanta .2019.01.040.

Zhou, Z., N. Hao, Y. Zhang, R. Hua, J. Qian, Q. Liu, H. N. Li, W. H. Zhu, and K. Wang. 2017. A novel universal colorimetric sensor for simultaneous dual targets detection through DNA-directed self-assembly of graphene oxide and magnetic separation. Chem. Commun. (Camb.) 53:7096-7099. https://doi.org/10.1039/ C7CC03914.J.

\section{ORCIDS}

Sihan Chen $\odot$ https://orcid.org/0000-0002-5249-8723

Chaoxin Man (ㄴ) https://orcid.org/0000-0003-2050-4639

Yujun Jiang @ https://orcid.org/0000-0002-2857-2137 